\title{
Therapeutic Challenges in Chronic Myeloid Leukemia: A Case-Based Discussion
}

\author{
Ariel Perez ${ }^{\mathrm{a}, \mathrm{c}}$, Grant Jester ${ }^{\mathrm{a}, \mathrm{c}}$, Yehuda Galilib, \\ Ahmad El-Far ${ }^{\text {a }}$, Said Baidas ${ }^{\text {a }}$
}

\begin{abstract}
Chronic myeloid leukemia (CML) is a myeloproliferative neoplasm characterized by a reciprocal translocation between the long arms of chromosomes 9 and 22 that results in expression of the oncoprotein BCR-ABL1. An optimal response to tyrosine kinase inhibitors (TKIs) requires a $B C R-A B L$ transcript level $\leq 10 \%$ at 3 months, $\leq$ $1 \%$ at 6 months, $\leq 0.1 \%$ at 1 year, and $\leq 0.01 \%$ onwards. Complex scenarios like $\mathrm{P} 190^{\mathrm{BCR}-\mathrm{ABL}} \mathrm{CML}$, unusual $B C R-A B L$ transcripts, primary refractory CML, and detection of TKI-resistance mutations during treatment frequently pose a therapeutic challenge. In this article we present some of these clinical scenarios using a case-based approach.
\end{abstract}

Keywords: CML; Unusual transcripts; TKI resistance

\section{Introduction}

Chronic myeloid leukemia (CML) is a myeloproliferative neoplasm characterized by a reciprocal translocation between the long arms of chromosomes 9 and 22, specifically $\mathrm{t}(9 ; 22)$ (q34.1; 11.2 ), which rearranges the Abelson murine leukemia viral oncogene homolog $1(A B L 1)$ and breakpoint cluster region $(B C R)$ genes [1]. In 2016, there were estimated 54,226 people with CML in the USA [2]. The estimated number of new cases for 2019 in the USA is 8,990 [2]. The course of CML can be divided in three phases: chronic, accelerated or blast phase [3]. Most patients being diagnosed are in the chronic phase.

The introduction of tyrosine kinase inhibitor (TKI) therapy changed the landscape of this disease. Long-term outcomes of imatinib-treated patients in the International Ran-

Manuscript submitted May 28, 2020, accepted June 11, 2020

Published online June 29, 2020

${ }^{a}$ UF Health Cancer Center at Orlando Health, Orlando, FL 32806, USA

bInternal Medicine Department, Orlando Health, Orlando, FL 32806, USA

${ }^{c}$ Corresponding Authors: Ariel Perez and Grant Jester, UF Health Cancer Center at Orlando Health, 1400 S. Orange Ave, MP \#700-4, Orlando, FL 32806,

USA. Emails: app1279@gmail.com and grant.jester@orlandohealth.com

doi: https://doi.org/10.14740/jmc3510 domized Study of Interferon and STI571 (IRIS) showed an overall survival rate of $83.3 \%$ at 10 years [4]. Achieving an optimal response requires $B C R-A B L$ transcripts level $\leq 10 \%$ at 3 months, $\leq 1 \%$ at 6 months, $\leq 0.1 \%$ at 1 year, and $\leq 0.01 \%$ onwards [5]. Resistance-associated mutations will develop in more than $30 \%$ of CML patients treated with TKI [6]. Most known resistance-associated mutations are simple point mutations that take place in the BCR-ABL1 kinase domain and result in ineffective drug binding [7]. Next-generation sequencing has allowed detection of leukemia-associated mutations in patients with TKI resistance in the absence of kinase domain mutations. For example, mutations in DNMT3A and ASXL1 could lead to TKI resistance [8]. Mutation mechanisms independent of $B C R-A B L 1$ are more likely seen in primary resistance [9].

High-complexity scenarios like molecular diagnostic issues, primary refractory CML, and emergence of TKI resistance-associated mutations frequently pose therapeutic challenges. In this article, we will discuss some of these clinical scenarios using a case-based approach.

\section{Case Reports}

\section{Case 1: primary imatinib resistance in $\mathrm{P} 190^{\mathrm{BCR}-\mathrm{ABL}} \mathrm{CML}$}

A 71-year-old male presented to our practice in November 2017 for incidental finding of persistent leukocytosis on laboratory studies. His complete blood count $(\mathrm{CBC})$ showed a total white blood cell (WBC) count of $22.3 \times 10^{3} / \mu \mathrm{L}$, hemoglobin $(\mathrm{Hb})$ of $12.9 \mathrm{~g} / \mathrm{dL}$, and platelets of $114 \times 10^{3} / \mu \mathrm{L}$. WBC differential showed $4 \%$ myelocytes and $2 \%$ metamyelocytes. A diagnostic bone marrow performed in January 2018 showed a markedly hypercellular marrow (almost 100\% cellularity) characterized by marked granulocytic hyperplasia consistent with a diagnosis of CML. Cytogenetics showed abnormal male karyotype with loss of $\mathrm{Y}$ chromosome and $\mathrm{t}(9 ; 22)$ detected in all cells examined. Quantitative reverse transcription-polymerase chain reaction (qRT-PCR) for $B C R$ $A B L 1$ on peripheral blood and marrow identified presence of $B C R-A B L 1$ fusion transcript e1a2 involving minor (p190) breakpoint. He was started on imatinib in mid-January 2018. $\mathrm{He}$ achieved a complete hematological response soon after initiation of TKI therapy, but his $B C R-A B L$ transcript levels over the following 6 months raised concerns for TKI-re- 


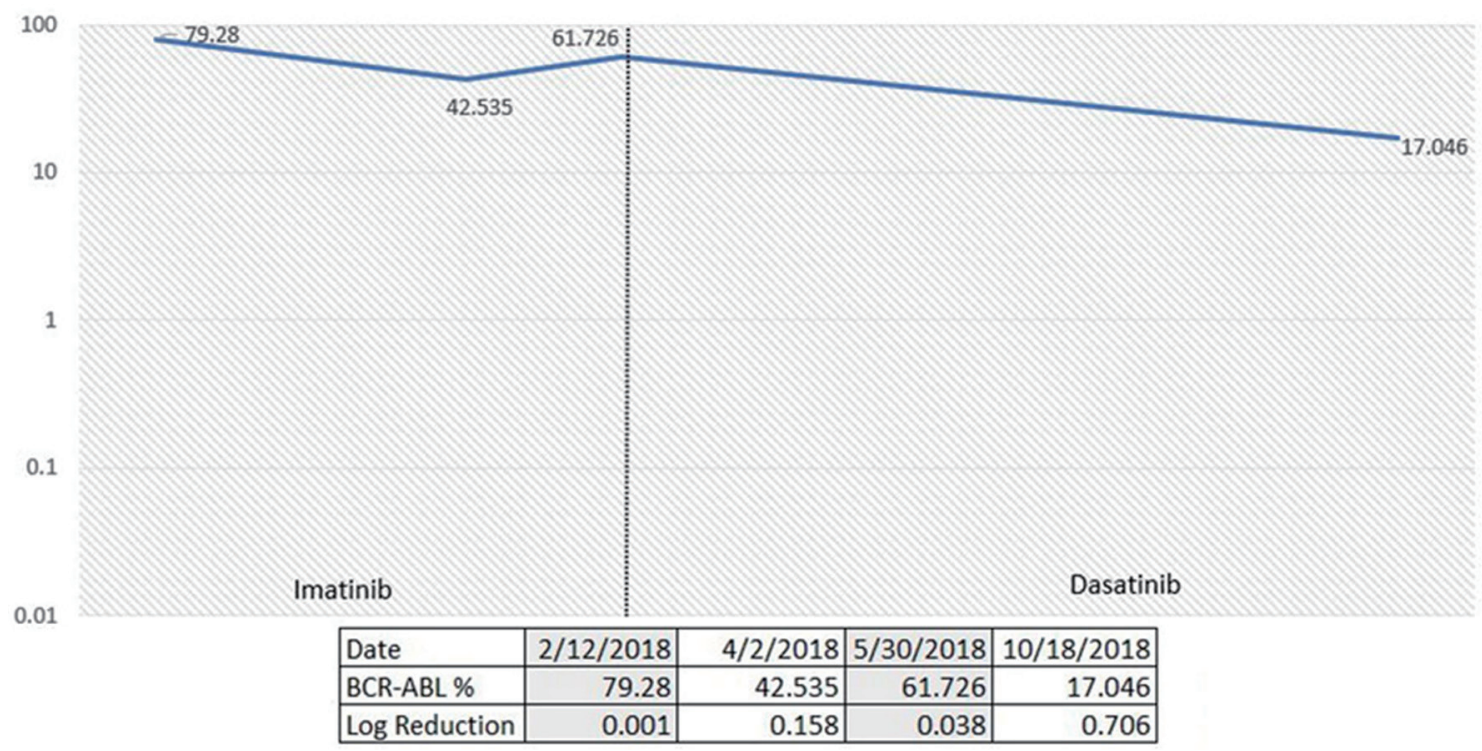

Figure 1. Percentage of $\mathrm{p} 190 B C R-A B L 1$ via quantitative reverse transcription-polymerase chain reaction (qRT-PCR) over time according to TKI choices. TKI: tyrosine kinase inhibitor.

sistant disease. ABL1 kinase mutation analysis did not show any resistance-associated mutations. He was switched to the second generation TKI therapy with dasatinib in June 2018. $B C R-A B L 1$ transcripts level by qRT-PCR on peripheral blood was $61.726 \%$ by international scale (IS) prior to dasatinib. Follow-up result in October 2018 was $17.046 \%$ by IS, with a $\log$ reduction of 0.706 . There was response to dasatinib noted at 3 months with more than $50 \%$ decrease from baseline levels (Fig. 1). Although he was still above the desired 10\% mark, we decided to continue treatment and revaluate again in 3 months. Unfortunately, the patient was lost to follow-up after that office visit.

\section{Discussion}

CML with isolated $B C R-A B L 1$ fusion transcript e1a2 involving minor (p190) breakpoint is extremely rare. In a MD Anderson Cancer Center study, P190 $190^{\mathrm{BCR}-\mathrm{ABL}}$ CML represented only $1 \%$ of patients with CML and it was associated with an inferior outcome to therapy with TKI [10]. P190 ${ }^{\mathrm{BCR}-\mathrm{ABL}} \mathrm{CML}$ is often associated with peripheral monocytosis, absence of splenomegaly and bone marrow morphologic features that are intermediate between CML and chronic myelomonocytic leukemia (CMML) [11]. It has also been associated with frequent additional cytogenetic abnormalities. A recent study presented at the 2019 American Society of Hematology meeting recommended P190 ${ }^{\mathrm{BCR}-\mathrm{ABL}}$ CML to be considered as a high-risk group. The authors from Helsinki University demonstrated that p190 activates specific cancer pathways, notably Src signaling and interferon pathways [12]. Management of these patients requires strict TKI response monitoring and early consideration of stem cell transplant in eligible patients. Frontline use of the second generation TKI in patients with $\mathrm{P} 190^{\mathrm{BCR}-\mathrm{ABL}} \mathrm{CML}$ should be strongly considered.

\section{Case 2: primary imatinib resistance with $A S X L 1$ and $D N$ - MT3A mutations}

A 37-year-old African American male was initially admitted to our center for persistent headache and fatigue. His CBC showed WBC of $323 \times 10^{3} / \mu \mathrm{L}, \mathrm{Hb}$ of $6.7 \mathrm{~g} / \mathrm{dL}$, and platelets of $1,120 \times 10^{3} / \mu \mathrm{L}$. WBC differential showed $28 \%$ myelocytes and $4 \%$ blasts. He underwent a diagnostic bone marrow biopsy in September 2017 which showed a markedly hypercellular marrow (100\% cellularity) with myeloid hyperplasia and increased atypical megakaryocytes. Chromosome analysis showed 46XY karyotype with translocation $(9 ; 22)$. BCR$A B L$ by qRT-PCR was detected at $42.369 \%$ by IS involving the major break point (p210). Molecular profiling showed ASXL1 and DNMT3A mutations. He was started on imatinib at time of hospitalization. He achieved a complete hematological response with imatinib, but no molecular response. ABL1 kinase mutation analysis was done in January 2018 with no mutations detected. He was then switched to dasatinib $100 \mathrm{mg}$ daily in May 2018 with eventual dose reduction to $70 \mathrm{mg}$ due to grade 3 gastrointestinal toxicity with diarrhea. He failed to achieve a molecular response to dasatinib. In January 2019 he was switched to ponatinib and referred for allogeneic stem cell transplant evaluation. $B C R-A B L 1$ transcript level by qRT-PCR prior to initiation of ponatinib was $38.848 \%$. Fluctuating transcript levels were attributed to issues with treatment adherence. Latest qRT-PCR results in December 2019 (11 months after ponatinib initiation) showed a $B C R-A B L 1$ level of $2.350 \%$ by IS with a $1.485 \log$ reduction (Fig. 2).

\section{Discussion}

Patients with CML diagnosed in the chronic phase can be tri- 


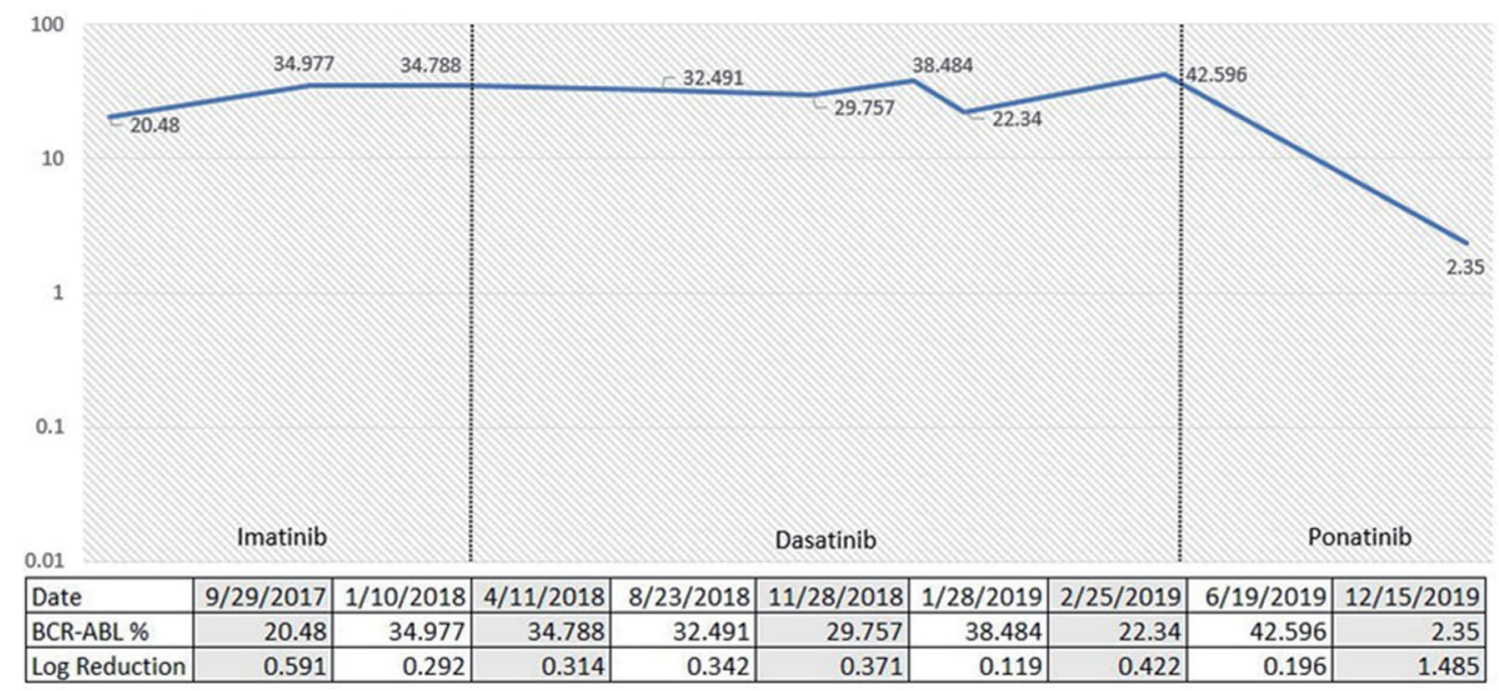

Figure 2. Percentage of p210 BCR-ABL1 via quantitative reverse transcription-polymerase chain reaction (qRT-PCR) over time according to $\mathrm{TKI}$ choices. TKI: tyrosine kinase inhibitor.

aged into initial TKI treatment choice based upon prognostic scoring systems, such as Sokal or European Treatment and Outcome Study (EUTOS), with higher risk patients generally being offered frontline second-generation TKIs [13]. Our patient was high risk based upon the Sokal system, but unfortunately was not a candidate for the second generation TKI due to cost and insurance issues. The patient achieved a complete hematologic response with imatinib but did not show any signs of molecular response with rising $B C R-A B L 1$ transcript levels. Primary imatinib failure in chronic phase CML is not an uncommon scenario, and patients in this category may still achieve a response to the second and third generation TKI with a $72 \%$ overall survival at 3 years [14]. Our patient was tested for ABL1 kinase mutation and found to be negative, which is seen in about $50 \%$ of patients who are non-responders to TKI medications [6]. Next-generation sequencing performed at the time of diagnosis revealed $A S X L 1$ and $D N M T 3 A$ mutations. It has been previously described that the development of clonal evolution is associated with decrease in both response and overall survival with the use of imatinib [15]. More recently there has been growing evidence that both $A S X L 1$ and $D N$ $M T 3 A$ mutations are associated with TKI resistance and progression to more advanced stages of CML [8]. Next-generation sequencing for common myeloid somatic mutations may be helpful to determine the risk of initial TKI failure in patients with chronic phase CML.

\section{Case 3: primary imatinib resistance with c.1424 35-NT mutation}

A 45-year-old female was admitted to our center in June 2017 with hyperleukocytosis. Her initial CBC showed WBC of 334 $\times 10^{3} / \mu \mathrm{L}, \mathrm{Hb}$ of $8.7 \mathrm{~g} / \mathrm{dL}$ and platelets of $268 \times 10^{3} / \mu \mathrm{L}$. WBC differential showed $23 \%$ myelocytes, $12 \%$ metamyelocytes, $3 \%$ promyelocytes, and $4 \%$ blasts. She underwent a diagnostic bone marrow in June 2017 showing a hypercellular marrow with 100\% cellularity. Cytogenetics showed 46XX karyotype with positive $t(9 ; 22)$. The FISH studies confirmed $t(9 ; 22)$. She had some delays in starting treatment due to financial barriers but was eventually started on imatinib $400 \mathrm{mg}$ once a day. She achieved a complete hematological response rapidly. However, she continued to have persistent detection of $B C R-A B L$ mutation at $12.7 \%$ with $\log$ reduction of 0.773 at 6 months (Fig. 3). A mutation analysis was performed showing an ABL kinase domain mutation, specifically a c.1424 35-NT insertion. By the end of January 2018, she was started on bosutinib 500 mg per day. The patient developed significant gastrointestinal toxicity and required a dose reduction to $400 \mathrm{mg}$ per day. She achieved a major molecular response 12 months after bosutinib initiation, which has sustained so far with latest qRT-PCR in July 2019 showing $0.016 \%$ by IS with log reduction of 3.571 (Fig. 3). The patient continued to have major molecular response after bosutinib dose reduction.

\section{Discussion}

This case highlights another instance of failure to achieve an adequate response with frontline imatinib. The patient was initially treated with imatinib with an increase in $B C R-A B L$ transcripts nearly two-fold from baseline. A prior study has indicated that two-fold rises in consecutive samples of $B C R-A B L$ transcripts predicts a greater than $60 \%$ possibility of identifying a kinase domain mutation [16]. The patient had ABL1 kinase mutation analysis that showed a c.1424 35-NT insertion. Point mutations are generally thought to confer resistance to imatinib therapy and nearly 100 different types have been documented [17]. Interestingly, this patient's point mutation has conflicting reports as to its contribution to TKI resistance. One group reported that a 35 -base pair insertion alters the tyrosine kinase in a manner similar to the T315I mutation [18]. Another group concluded that the $35-\mathrm{NT}$ insertion did not correlate with TKI resistance [19]. The patient was ultimately 


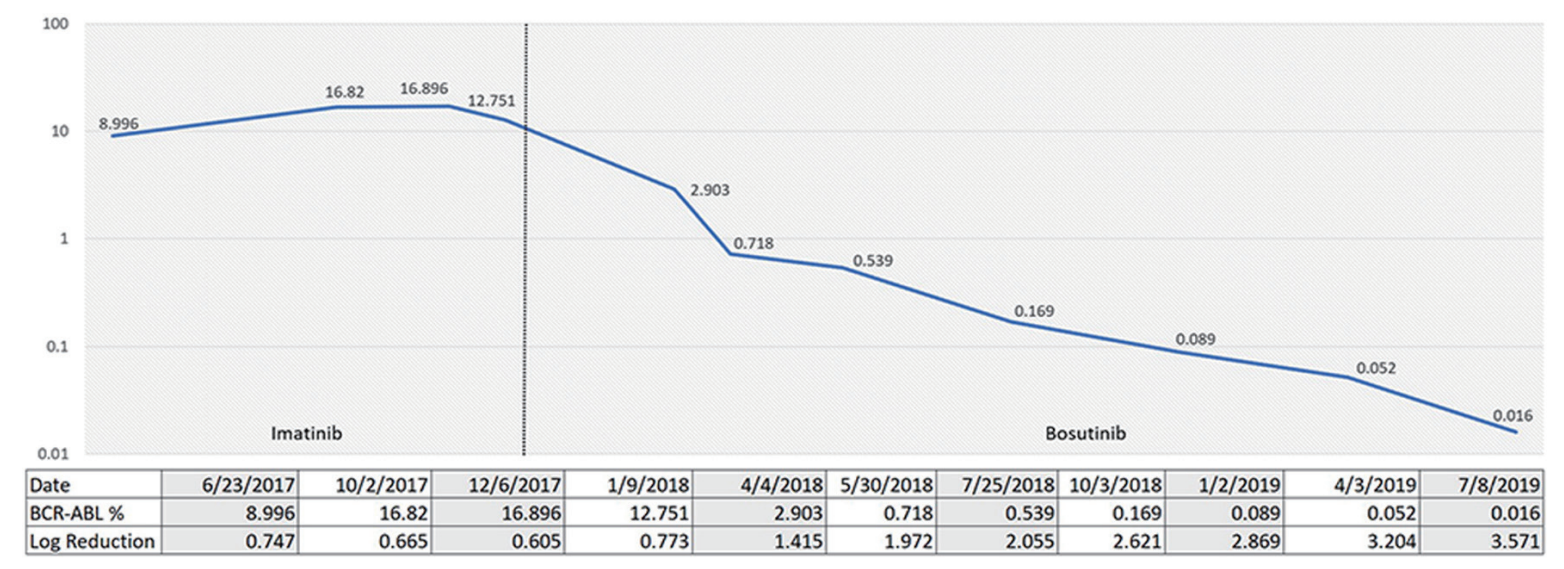

Figure 3. Percentage of p210 BCR-ABL1 via quantitative reverse transcription-polymerase chain reaction (qRT-PCR) over time according to TKI choices. TKI: tyrosine kinase inhibitor.

transitioned to bosutinib with an excellent response achieving a major molecular response (MMR). Our case demonstrates that 35-NT insertions can lead to imatinib resistance and may respond to the second generation TKIs.

\section{Case 4: positive fluorescence in situ hybridization (FISH) for $\mathrm{t}(9 ; 22)$ with negative RT-PCR for $B C R-A B L 1$}

A 72-year-old female initially presented to our center for evaluation of leukocytosis. Her CBC showed WBC of $73 \times 10^{3} / \mu \mathrm{L}$, $\mathrm{Hb}$ of $12 \mathrm{~g} / \mathrm{dL}$ and platelets of $464 \times 10^{3} / \mu \mathrm{L}$. WBC differential showed $3 \%$ myelocytes, $14 \%$ metamyelocytes and $1 \%$ blasts. The cytogenetic analysis on peripheral blood showed an abnormal female karyotype, with $\mathrm{t}(9 ; 22)$ on all cells examined. FISH analysis confirmed $\mathrm{t}(9 ; 22)$ in $95.5 \%$ of analyzed nuclei (normal $<1.5 \%$ ). $B C R-A B L$ by PCR was negative, including standard p210, p190 testing and non-standard BCR-ABL PCR testing for $\mathrm{p} 230$. She was started on the frontline treatment with imatinib in July 2018. She achieved a complete morphologic response but soon after developed grade 3 skin rash and was unable to tolerate imatinib further. She was then switched to dasatinib. FISH analysis on peripheral blood was reported as negative in February 2019 (6 months after initiation of therapy). FISH has remained undetectable since then, except for a one-time low level positive at $2 \%$ during a temporary TKItreatment interruption. She has been reluctant to have a bone marrow biopsy performed from time of initial diagnosis. We have provided frequent education regarding its importance for morphological evaluation, and for the subsequent assessment of cytogenetic response. Most recent peripheral blood FISH in September 2019 remains undetectable.

\section{Discussion}

Peripheral blood studies for detection of $\mathrm{t}(9 ; 22)$ or $B C R-A B L 1$ are not considered enough for diagnosis of CML. Bone marrow aspiration and biopsy is required for all patients in whom CML is suspected. It will confirm the diagnosis and provide information needed for staging in terms of the blast and basophil percentages [20]. Our patient was informed in detail of the rationale behind a diagnostic bone marrow examination, but she still refused. To further complicate the situation, although cytogenetics and FISH on peripheral blood were both positive, molecular testing for $B C R-A B L 1$ transcripts by RTPCR was negative making response assessment challenging. Possible reasons for discordance between FISH and RT-PCR testing were discussed with molecular pathologist. Standard commercial testing included reverse transcription of extracted sample ribonucleic acid (RNA) to complementary deoxyribonucleic acid (cDNA) for $B C R-A B L 1$ fusions e13a2, e14a2 (p210) and ela2 (p190). Additional non-standard testing for e19a2 (p230) was also performed. A false-negative PCR result was considered initially, but repeated testing was consistently negative making sampling or procedural errors unlikely. We believe the discordant results in this case could be explained by the presence of an atypical fusion transcript. Rare breakpoints having junction configurations other than e13a2, e14a2, e1a2 or e19a2 have been reported [21]. Management of these cases is challenging, especially when access to advanced molecular testing is limited. Most practices rely on standardized send-out testing using commercially available products. After extensive discussion with the patient, it was agreed to initiate TKI therapy and to monitor using FISH on peripheral blood. For correlative purposes, a complete cytogenetic response was considered equivalent to a negative FISH test $( \pm 2 \%)$, given the lack of a marrow examination [20].

\section{Conclusions}

Upfront treatment with imatinib although attractive from a cost benefit standpoint has limitations for selected patients with unusual $B C R-A B L 1$ transcripts or presence of specific mutations. The second generation TKIs are appropriate in these situations as frontline therapy. Close monitoring, transition to ponatinib if no response to the second generation TKIs and consideration of bone marrow transplant evaluation in eligible patients is recommended. 


\section{Acknowledgments}

None to declare.

\section{Financial Disclosure}

No funding was received. None of the authors have disclosures relevant to this manuscript.

\section{Conflict of Interest}

None to declare.

\section{Informed Consent}

Not applicable. The manuscript has been sufficiently de-identified to protect the patients.

\section{Author Contributions}

AP and GJ wrote the manuscript and have joint first authorship. YG and AP designed the figures. All authors contributed to the editing of the manuscript. All authors have reviewed the final version and approved it for publication. SB developed the idea and revised the manuscript as the senior author.

\section{Data Availability}

The authors declare that data supporting the findings of this study are available within the article.

\section{References}

1. Rowley JD. Letter: A new consistent chromosomal abnormality in chronic myelogenous leukaemia identified by quinacrine fluorescence and Giemsa staining. Nature. 1973;243(5405):290-293.

2. Chronic Myeloid Leukemia - Cancer Stat Facts. SEER. https://seer.cancer.gov/statfacts/html/cmyl.html. Accessed on Oct 3, 2020.

3. Arber DA, Orazi A, Hasserjian R, Thiele J, Borowitz MJ, Le Beau MM, Bloomfield CD, et al. The 2016 revision to the World Health Organization classification of myeloid neoplasms and acute leukemia. Blood. 2016;127(20):2391-2405.

4. Hochhaus A, Larson RA, Guilhot F, Radich JP, Branford S, Hughes TP, Baccarani M, et al. Long-term outcomes of imatinib treatment for chronic myeloid leukemia. N Engl J Med. 2017;376(10):917-927.

5. Baccarani M, Castagnetti F, Gugliotta G, Rosti G. A review of the European LeukemiaNet recommendations for the management of CML. Ann Hematol. 2015;94(Suppl 2):S141-147.

6. Jabbour E, Kantarjian H, Jones D, Talpaz M, Bekele N, O'Brien S, Zhou X, et al. Frequency and clinical significance of BCR-ABL mutations in patients with chronic myeloid leukemia treated with imatinib mesylate. Leukemia. 2006;20(10):1767-1773.

7. Patel $\mathrm{AB}, \mathrm{O}^{\prime}$ Hare $\mathrm{T}$, Deininger MW. Mechanisms of resistance to $\mathrm{ABL}$ kinase inhibition in chronic myeloid leukemia and the development of next generation ABL kinase inhibitors. Hematol Oncol Clin North Am. 2017;31(4):589-612.

8. Kim TS, Zhang Z, Tyndel M, Ahn J-S, Choi S, Kim HJ, Lipton $\mathrm{JH}$, et al. Whole exome sequencing reveals that DNMT3A and ASXL1 mutation are involved in the development of tyrosine kinase inhibitor resistance in chronic myeloid leukemia patients without ABL1 tyrosine kinase domain mutation. Blood. 2015;126(23):1568.

9. Yaghmaie M, Yeung CC. Molecular Mechanisms of Resistance to Tyrosine Kinase Inhibitors. Curr Hematol Malig Rep. 2019;14(5):395-404.

10. Verma D, Kantarjian HM, Jones D, Luthra R, Borthakur G, Verstovsek S, Rios MB, et al. Chronic myeloid leukemia (CML) with P190 BCR-ABL: analysis of characteristics, outcomes, and prognostic significance. Blood. 2009;114(11):2232-2235.

11. Melo JV, Myint H, Galton DA, Goldman JM. P190BCR-ABL chronic myeloid leukaemia: the missing link with chronic myelomonocytic leukaemia? Leukemia. 1994;8(1):208-211.

12. Awad SA, Hohtari H, Javarappa KK, Brandstoetter T, Kim D, Potdar S, Heckman CA, et al. BCR-ABL1 p190 in CML: a minor breakpoint with a major impact. Blood. 2019;134(Suppl_1):190.

13. Cortes JE. A second-generation TKI should always be used as initial therapy for CML. Blood Adv. 2018;2(24):36533655.

14. Kantarjian H, O'Brien S, Talpaz M, Borthakur G, Ravandi F, Faderl S, Verstovsek S, et al. Outcome of patients with Philadelphia chromosome-positive chronic myelogenous leukemia post-imatinib mesylate failure. Cancer. 2007;109(8):1556-1560.

15. Apperley JF. Part I: mechanisms of resistance to imatinib in chronic myeloid leukaemia. Lancet Oncol. 2007;8(11):1018-1029.

16. Soverini S, Hochhaus A, Nicolini FE, Gruber F, Lange T, Saglio G, Pane F, et al. BCR-ABL kinase domain mutation analysis in chronic myeloid leukemia patients treated with tyrosine kinase inhibitors: recommendations from an expert panel on behalf of European LeukemiaNet. Blood. 2011;118(5):1208-1215.

17. Branford S, Rudzki Z, Parkinson I, Grigg A, Taylor K, Seymour JF, Durrant S, et al. Real-time quantitative PCR analysis can be used as a primary screen to identify patients with CML treated with imatinib who have BCRABL kinase domain mutations. Blood. 2004;104(9):29262932.

18. Lee TS, Ma W, Zhang X, Giles F, Cortes J, Kantarjian $\mathrm{H}$, Albitar M. BCR-ABL alternative splicing as a com- 
mon mechanism for imatinib resistance: evidence from molecular dynamics simulations. Mol Cancer Ther. 2008;7(12):3834-3841.

19. O'Hare T, Zabriskie MS, Eide CA, Agarwal A, Adrian LT, You H, Corbin AS, et al. The BCR-ABL35INS insertion/ truncation mutant is kinase-inactive and does not contribute to tyrosine kinase inhibitor resistance in chronic my- eloid leukemia. Blood. 2011;118(19):5250-5254

20. Jabbour E, Kantarjian H. Chronic myeloid leukemia: 2018 update on diagnosis, therapy and monitoring. Am J Hematol. 2018;93(3):442-459.

21. Barnes DJ, Melo JV. Cytogenetic and molecular genetic aspects of chronic myeloid leukaemia. Acta Haematol. 2002;108(4):180-202. 